OPEN ACCESS

Edited by:

Shisan Bao,

The University of Sydney, Australia

Reviewed by:

Susanta Kumar Ghosh,

National Institute of Malaria Research

(ICMR), India

Guilherme Dias de Melo,

Institut Pasteur, France

*Correspondence:

Marcos Alejandro Mayer

marcos.mayer@gmail.com

tThese authors have contributed equally to this work

Specialty section:

This article was submitted to Infectious Diseases - Surveillance,

Prevention and Treatment,

a section of the journal

Frontiers in Public Health

Received: 11 November 2021

Accepted: 31 January 2022

Published: 22 February 2022

Citation:

Mayer MA, Krolewiecki A, Ferrero A, Bocchio M, Barbero J, Miguel M, Paladini A, Delgado C, Ojeda JR,

Elorza $C$, Bertone A, Fleitas $P E$, Vera $G$ and Kohan MR (2022) Safety and Efficacy of a MEURI Program for the Use of High Dose Ivermectin in COVID-19 Patients.

Front. Public Health 10:813378. doi: 10.3389/fpubh.2022.813378

\section{Safety and Efficacy of a MEURI Program for the Use of High Dose Ivermectin in COVID-19 Patients}

\author{
Marcos Alejandro Mayer ${ }^{1,2,3 *}$, Alejandro Krolewiecki ${ }^{3,4 \dagger}$, Alejandro Ferrero ${ }^{1}$, \\ Marcelo Bocchio ${ }^{1}$, Juan Barbero ${ }^{1}$, Marcos Miguel ${ }^{1}$, Ariel Paladini ${ }^{1}$, Carlos Delgado ${ }^{1}$, \\ Juan Ramón Ojeda ${ }^{1}$, Claudia Elorza ${ }^{1}$, Ana Bertone ${ }^{1}$, Pedro Emanuel Fleitas ${ }^{3,4}$, \\ Gustavo Vera ${ }^{1}$ and Mario Rubén Kohan ${ }^{1}$

\begin{abstract}
${ }^{1}$ Ministerio de Salud de la Provincia de La Pampa, Santa Rosa, Argentina, ${ }^{2}$ Fundación Centro de Salud e Investigaciones Médicas, Santa Rosa, Argentina, ${ }^{3}$ Consejo Nacional de Investigaciones Científicas y Técnicas, Buenos Aires, Argentina, ${ }^{4}$ Instituto de Investigaciones de Enfermedades Tropicales, Universidad Nacional de Salta, Sede Regional Orán, Salta, Argentina
\end{abstract}

Background: In the absence of antiviral alternatives, interventions under research for COVID-19 might be offered following guidelines from WHO for monitored emergency use of unregistered and experimental interventions (MEURI). Ivermectin is among several drugs explored for its role against SARS-CoV-2, with a well-known safety profile but conflicting data regarding clinical utility for COVID-19. The aim of this report is to inform on the results of a MEURI Program of high-dose ivermectin in COVID-19 carried out by the Ministry of Health of the Province of La Pampa, Argentina.

Methods: COVID-19 subjects, within 5 days of symptoms onset were invited to participate in the program, which consisted in the administration of ivermectin 0.6 $\mathrm{mg} / \mathrm{kg} /$ day for 5 days plus standard of care. Active pharmacosurveillance was performed for 21 days, and hepatic laboratory assessments were performed in a subset of patients. Frequency of Intensive Care Unit (ICU) admission and COVID-19-related mortality of subjects in the ivermectin intention to treat group were compared with that observed in inhabitants of the same province during the same period not participating in the program.

Results: From 21,232 subjects with COVID-19, 3,266 were offered and agreed to participate in the ivermectin program and 17,966 did not and were considered as controls. A total of 567 participants reported 819 adverse events (AEs); 3.13\% discontinued ivermectin due to adverse events. ICU admission was significantly lower in the ivermectin group compared to controls among participants $\geq 40$ year-old ( $1.2 \mathrm{vs}$. $2.0 \%$, odds ratio $0.608 ; p=0.024)$. Similarly, mortality was lower in the ivermectin group in the full group analysis ( $1.5 \mathrm{vs.} 2.1 \%$, odds ratio $0.720 ; p=0.029$ ), as well as in subjects $\geq 40$ year- old ( 2.7 vs. $4.1 \%$, odds ratio $0.655 ; p=0.005$ ).

Conclusions: This report highlights the safety and possible efficacy of high dose ivermectin as a potentially useful intervention deserving public health-based consideration for COVID-19 patients.

Keywords: ivermectin, COVID-19, mortality, ICU-admission, safety 


\section{INTRODUCTION}

COVID-19 constitutes a public health emergency at a global scale since its appearance in Wuhan, China, in December 2019 (1). By the end of January 2022, over 360 million cases and 5.5 million deaths have been reported worldwide (2). Vaccine rollout campaigns, which currently offer the best hopes for pandemic control, are a key targeted pharmacologic intervention for containment of disease spread and impact on the incidence of severe cases $(3,4)$.

Despite having an asymptomatic or mild course in most cases, COVID-19 constitutes a significant burden on health systems unprepared to cope with outbreaks requiring, among other things, massive testing capacity for rapid case detection and isolation, expansion of intensive care unit (ICU) capacity and case management guidelines for a previously unknown pathogen. This public health crisis has been, and still is, more profound in countries with weaker health systems (5).

The unprecedented progress in vaccine development has not been matched by the development of antiviral molecules, either new or repurposed, that could contribute to the treatment or prevention of COVID-19. With convalescent plasma, monoclonal antibodies, hydroxychloroquine and antiretrovirals among many molecules tested in-vitro and in observational and clinical trials, different treatment guidelines only agree in the use of corticosteroids, thromboprofilaxis and respiratory support in their recommendations $(6,7)$. In terms of antivirals, remdesivir has been incorporated in some treatment guidelines and more recently, some have incorporated newly developed antiviral drugs like ritonavir-boosted nirmatrelvir, sotrovimab, and molnupiravir as treatment alternatives for high-risk nonhospitalized patients based on the results of clinical trials (8).

Ivermectin (IVM) is an endectocide drug widely used for the treatment and control of onchocerciasis and lymphatic filariasis through mass drug administration programs, which has a wide therapeutic index and a benign safety profile in the currently approved doses of $150-400 \mu \mathrm{g} / \mathrm{kg}$ mostly in single dose regimens $(9,10)$. Besides its known uses, it has been evaluated as an antiviral, demonstrating in vitro activity against zika, rabies and dengue among other viruses (11). In the case of dengue, a recently published randomized clinical trial from Thailand showed positive although inconclusive results (12). For SARS-CoV-2, early on the pandemic, the report of the antiviral activity of IVM in Vero cells cultures sparked widespread interest in the potential utility of this oral, safe and affordable drug against COVID-19 (13). However, after over a year of several publications addressing this question, there is a lack of clear evidence for or against the use of IVM in COVID-19 patients $(6,14)$. With at least two completed double-blind randomized clinical trials (RCTs) showing no effect in clinical endpoints, other smaller randomized trials using higher doses identified significant antiviral effects (15-19) or a reduction in the clinical signs, including anosmia (20). That undefined landscape is summarized by the current NIH COVID19 treatment Guidelines stating that there is insufficient data to recommend either for or against the use of IVM in COVID-19 patients (6).
In 2016, the World Health Organization (WHO) issued the Guidance for Managing Ethical Issues in Infectious Diseases Outbreaks, with the aim of complementing existing guidance on ethics in public health in situations of great uncertainty and including recommendations for the use of unproven interventions outside clinical trials, which based on a WHO response developed in the context of the outbreak of Ebola Virus Disease in Western Africa in 2014 are called "monitored emergency use of unregistered and experimental interventions" (MEURI) (21). These interventions apply when no proven effective treatments exist, it is not possible to initiate clinical trials immediately, existing preliminary data supports the intervention, relevant regulatory, ethical and scientific authorities approve such use, resources are available to minimize risks and patient's informed consent is obtained. Proper monitoring and timely sharing of the results with the wider medical and scientific community are also requirements to MEURI activities.

The aim of this report is to inform about the satisfactory safety and efficacy results of a MEURI Program for the use of high dose IVM in COVID-19 patients, carried out by the Ministry of Health of the Province of La Pampa, in the Patagonian region of Argentina.

\section{METHODS}

\section{MEURI Program for the Use of High Dose IVM in COVID-19 Patients}

By the end of January 2021, the Ministry of Health of the Province of La Pampa (Argentina) authorized the implementation of a MEURI program based on the use of high dose IVM (600 $\mu \mathrm{g} / \mathrm{kg}$ for 5 days) in COVID-19 adult patients (older than 18 year-old). In order to be able to participate, subjects had to be able to provide written informed consent, have a confirmed diagnosis of COVID-19 infection (by means of RT-PCR or antigen test) and symptoms onset within 5 days before entering the program. Exclusion criteria included pregnancy, breast feeding, hypersensitivity to IVM or acute allergic states, and active use of warfarin. Women with child-bearing potential were eligible if they were taking effective contraceptive measures before entering the program and agreed to continue with these measures for at least 30 days after receiving the last dose of IVM. Meanwhile ambulatory and inpatient subjects were allowed to participate (as long as they accomplished all inclusion criteria and had no exclusion criteria), admission to ICU was considered an exclusion criteria. Every member of the health staff of the Province of La Pampa was instructed to invite to participate in the program to every COVID-19 patient identified within the first 5 days of symptoms onset. However, participation in the program was voluntary, and physicians could decide not to include subjects in the program based on their medical criteria.

Ethical approval was obtained from the Provincial Ethics Committee of La Pampa, and participating individuals provided written informed consent. The study protocol is registered at national registry from Argentina (RENIS), with the registry number IS003403. 


\section{Intervention}

Participants were evaluated at program entry with complete medical history and a brief physical exam. At the beginning of the program, safety laboratory assessments before and at the end of treatment were mandatory. However, after a preliminary safety analysis that triggered an amendment approved by the Ethics Committee, these assessments were no longer mandatory and could be performed or not, based on medical judgment.

Patients received oral treatment with IVM for 5 consecutive days within $30 \mathrm{~min}$ of food ingestion, preferentially of high fat content, at $\sim 24 \mathrm{~h}$ intervals. IVM 6,9 , and $18 \mathrm{mg}$ tablets were used, combined to in all cases at a dose of $0.6 \mathrm{mg} / \mathrm{kg} /$ day based on baseline weight rounding to the lower full $(6 \mathrm{mg})$ dose. There were no specific guidelines regarding medical management of COVID-19 infection for the participants in the IVM program, which was the same as for the rest of the population. Standard of care in the Province of La Pampa at that time included no other antivirals and the use of systemic corticosteroids and deepvein thrombosis prophylaxis for hospitalized cases requiring oxygen supplementation to maintain oxygen saturation $\geq 94 \%$; for ambulatory cases, no disease specific interventions were included in the standard of care.

\section{Safety Assessment}

Active pharmacosurveillance was performed during the first 21 days after treatment start by means of the completion of a follow up chart, and safety assessment was based in all subjects that participated in the program in which follow up safety data was reported.

\section{Hepatic Safety Assessment}

Hepatic safety assessment was based on the analysis of hepatic lab exams performed before and after IVM treatment in a subset of patients, and consisted of laboratory determinations of alanine aminotransferase (ALT), aspartate aminotransferase (AST), alkaline phosphatase (ALP), and total bilirubin levels. Drug induced liver injury was defined according to the Latin American Association for Study of the liver definition, that includes (i) ALT elevation $\geq 5 \mathrm{ULN}$, (ii) ALP elevation $\geq 2 \mathrm{ULN}$ (in the absence of known bone pathology driving the increase in ALP level), or (iii) ALT $\geq 3$ ULN and simultaneous elevation of total bilirubin concentration above 2 ULN (22).

\section{Efficacy Analysis}

In order to estimate the efficacy of the implementation of the program, the clinical evolution of the subjects in the IVM intention to treat (ITT) group was compared with that observed in inhabitants of the same province during the same analyzed period (from January 20, 2021 to May 20 2021) who did not participate in the program (control group, C). To identify them, the analysis of the National Health Surveillance System (SNVS 2.0) was used, which records, among other events, the notification of COVID-19 cases, their clinical and demographic characteristics and the respective laboratory studies, in a mandatory, nominal and immediate way, according to a national regulation.
Given that the registration methodology differs between the one used in the IVM-monitored intervention program and the one used for registering subjects and events in SNVS 2.0 database, it was decided to consider variables not dependent on the registration method in the system for the efficacy analysis. Specifically, the primary objectives of the evaluation were the analysis of the impact of the program on the frequency of ICU admission and COVID-19-related death. It should be noted that both ICU admission and death registration is carried out centrally, so their identification is independent of the type of follow-up carried out.

In order to compare the clinical course of both groups, subjects under 18 years of age and pregnant women were excluded from the analysis. Also, as part of a bias control strategy, a sub-analysis was performed after excluding subjects with neoplastic diseases.

In the univariate efficacy analysis, subgroup analysis was performed according to age group (whole sample, subjects between 18 and 40 year old or subjects $\geq 40$ year old), immunization status (excluding subjects with at least one vaccine dose) and mean IVM prescribed dose.

\section{Statistical Analysis}

Baseline characteristics of the two groups (C and IVM) were compared by means of Student's T-test and Chi square. The clinical evolution was evaluated by Chi square Test and logistic regression analysis. Whenever possible, number needed to treat (NNT) values for IVM were estimated for the end points of ICU admission and death. The NNT values were estimated as the inverse of the difference in estimated absolute risk between control and IVM groups. In all cases, $p$-values < 0.05 were considered statistically significant. All analysis were performed with GraphPad Prism version 9.1.0 for Windows (La Jolla, California).

\section{RESULTS}

\section{Recruitment}

A total of 21,232 non-pregnant adults were identified as COVID19 positive between January 202021 and May 20 2021. Of these, 3,266 agreed to participate in the program and received at least one dose of IVM, and were included in the ITT analysis group. A group of 17,966 subjects that did not participate in the program were included in the $\mathrm{C}$ group. Descriptive characteristics of the population are presented in Table 1.

Safety follow up data was obtained from 2,613 subjects that participated in the program and were included in the Safety Analysis Group. Of these, 1,022 were followed with posttreatment hepatic lab exams, and were included in the Hepatic Safety Analysis group (Figure 1).

\section{Safety Analysis}

A total of 2,613 participants were included in the safety assessment. Five hundred sixty seven (567) participants reported 819 adverse events (AEs). Eighty-two subjects (3.13\%) discontinued IVM due to adverse events and all AEs resolved after treatment discontinuation. The most common AEs were 
TABLE 1 | Descriptive characteristics of the sample.

\begin{tabular}{|c|c|c|c|c|c|c|}
\hline \multirow[t]{2}{*}{ Variable } & \multicolumn{3}{|c|}{ Control group } & \multicolumn{3}{|c|}{ Ivermectin group } \\
\hline & $\begin{array}{c}\text { Total sample } \\
(n=17,966)\end{array}$ & $\begin{array}{c}18-40 \text { year old } \\
\text { subjects } \\
(n=8,944)\end{array}$ & $\begin{array}{c}\text { Subjects } \geq 40 \\
\text { year old } \\
(n=9,022)\end{array}$ & $\begin{array}{c}\text { Total sample } \\
(n=3,266)\end{array}$ & $\begin{array}{c}18-40 \text { year old } \\
\text { subjects } \\
(n=1,415)\end{array}$ & $\begin{array}{c}\text { Subjects } \geq 40 \\
\text { year old } \\
(n=1,851)\end{array}$ \\
\hline Age (years $\pm S D$ ) & $42.3 \pm 16.6$ & $28.6 \pm 6.1$ & $55.8 \pm 12.1$ & $43.8 \pm 15.4^{\star \star}$ & $29.8 \pm 5.9^{\star \star}$ & $54.4 \pm 11.5^{\star \star}$ \\
\hline \multirow[t]{2}{*}{ Sex } & $52.8 \%$ Female & $52.8 \%$ Female & $52.9 \%$ Female & 50.6\% Female & $50.1 \%$ Female & 47.18\% Female \\
\hline & $47.2 \%$ Male & $47.2 \%$ Male & $47.1 \%$ Male & 49.4\% Male & 49.9\% Male & 48.9\% Male \\
\hline Complete immunization & $323(1.8 \%)$ & $84(0.9 \%)$ & $239(2.6 \%)$ & $59(1.8 \%)$ & $22(1.6 \%)^{\star}$ & 37 (2.0\%) \\
\hline Incomplete immunization & $1,417(8.0 \%)$ & $97(1.1 \%)$ & $1,320(15 \%)$ & $271(8.5 \%)$ & $13(0.9 \%)$ & $258(14.2 \%)$ \\
\hline Cardiovascular disease & $1,792(10.0 \%)$ & $170(1.9 \%)$ & 1,622 (18.0\%) & $344(10.5 \%)$ & $29(2.0 \%)$ & $315(17.0 \%)$ \\
\hline COPD & 1,177 (6.6\%) & 384 (4.3\%) & $793(8.8 \%$ & 244 (7.5\%) & $73(5.2 \%)$ & $171(9.2 \%)$ \\
\hline Hypertension & $1,583(8.8 \%)$ & $67(0.7 \%)$ & $1,516(16.8 \%)$ & $551(16.9 \%)^{\star \star}$ & $27(1.9 \%)^{\star \star}$ & $524(28.3 \%)^{\star \star}$ \\
\hline Diabetes & $1,579(8.8 \%)$ & $209(2.3 \%)$ & $1,370(15.2 \%)$ & $315(9.6 \%)$ & $52(3.7 \%)^{\star \star}$ & $263(14.2 \%)$ \\
\hline Neoplasm & $269(1.5 \%)$ & $28(0.3 \%$ & $241(2.7 \%$ & 63 (1.9\%) & $10(0.7 \%)^{\#}$ & 53 (2.9\%) \\
\hline Obesity & $2,241(12.5 \%)$ & $760(8.5 \%)$ & $1,481(16.4 \%)$ & $1,182(36.2 \%)^{\star \star}$ & $401(28.3 \%)^{\star \star}$ & $781(42.2 \%)^{\star \star}$ \\
\hline
\end{tabular}

COPD, Chronic obstructive pulmonary disease.

Complete immunization: subjects with complete vaccine scheme at least 14 days before symptoms onset.

Incomplete immunization: subjects with the first vaccine dose (of a two-dose scheme) received at least 14 days before symptoms onset.

$\# p<0.05$ vs. control group.

${ }^{*} p<0.01$ vs. control group.

${ }^{* *} p<0.001$ vs. control group.

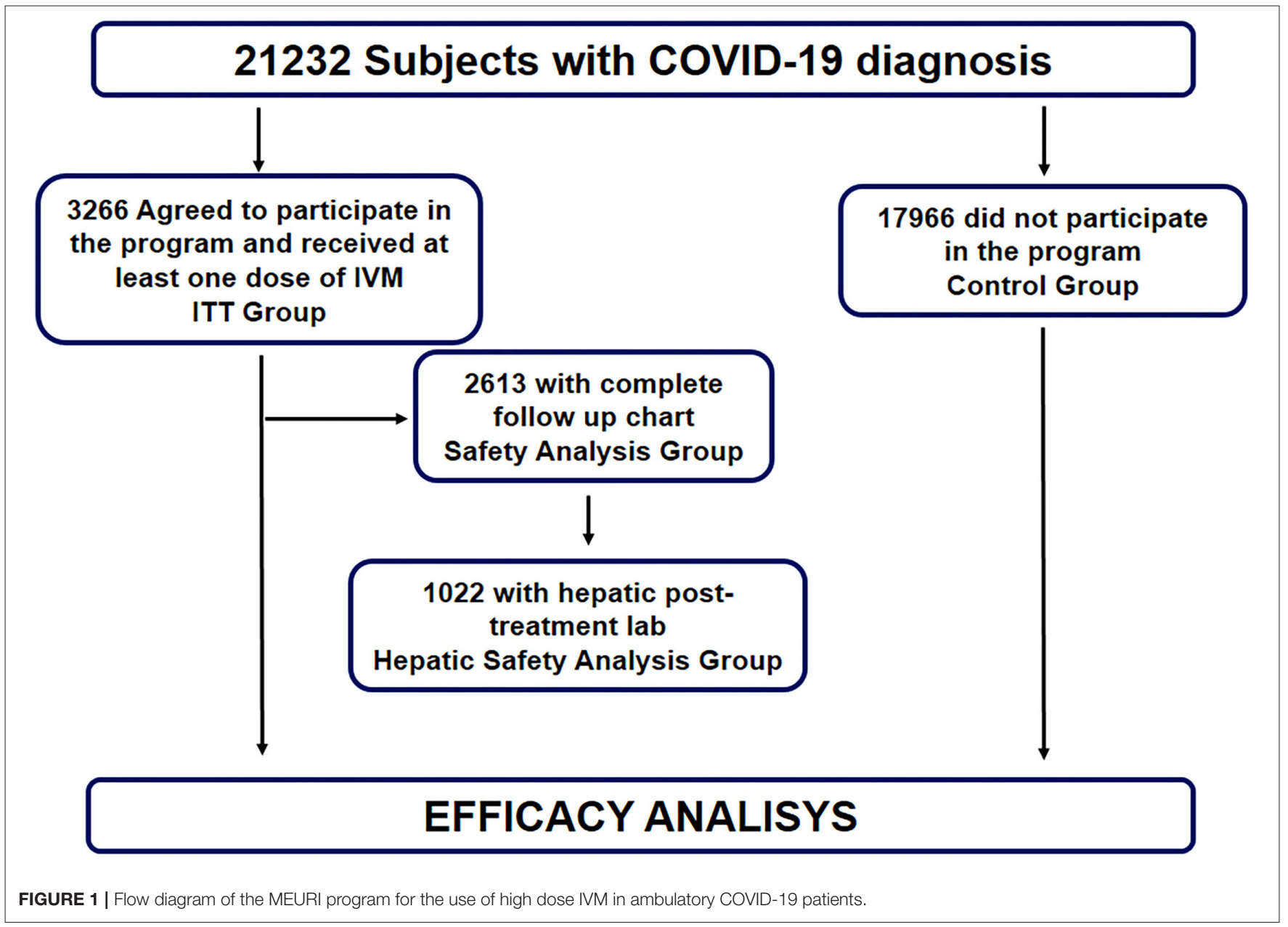


TABLE 2 | Safety analysis among subjects receiving ivermectin (n 2,613).

\begin{tabular}{lc}
\hline Adverse event & $\boldsymbol{N}(\%)$ \\
\hline Diarrhea & $155(5.93 \%)$ \\
Visual disorders & $136(5.2 \%)$ \\
Dizziness & $120(4.59 \%)$ \\
Abdominal pain & $91(3.48 \%)$ \\
Headache & $73(2.79 \%)$ \\
Nausea & $78(2.98 \%)$ \\
Anorexia & $31(1.19 \%)$ \\
Vomiting & $30(1.15 \%)$ \\
Heart rate elevation & $18(0.69 \%)$ \\
Rash & $16(0.61 \%)$ \\
Blood pressure elevation & $15(0.57 \%)$ \\
Pruritus & $14(0.53 \%)$ \\
Insomnia & $14(0.53 \%)$ \\
Drowsiness & $14(0.53 \%)$ \\
\hline
\end{tabular}

The table shows adverse events reported in more than $0.5 \%$ of subjects.

TABLE 3 | Hepatic safety analysis.

\begin{tabular}{lcc}
\hline Variable & Pre-treatment & Post-treatment \\
\hline AST $(n=1,000)$ & $34.07 \pm 29.59 \mathrm{U} / \mathrm{l}$ & $36.15 \pm 31.35 \mathrm{U} / /^{\star \star}$ \\
ALT $(n=988)$ & $25.67 \pm 14.93 \mathrm{U} / \mathrm{l}$ & $27.61 \pm 25.07 \mathrm{U} / /^{\star \star}$ \\
Total bilirrubin $(N=966)$ & $0.44 \pm 0.24 \mathrm{mg} / \mathrm{dl}$ & $0.47 \pm 0.24 \mathrm{mg} / \mathrm{dl}^{\star \star}$ \\
Alkaline phosphatase $(n=1,000)$ & $146.32 \pm 77.27 \mathrm{U} / \mathrm{l}$ & $120.60 \pm 93.33 \mathrm{U} / /^{\star \star}$ \\
\hline
\end{tabular}

${ }^{\star \star} p<0.001$ vs. pre-treatment values.

diarrhea, followed by visual disorders, dizziness, abdominal pain, headache and nausea (Table 2). Although many of the symptoms presented during treatment resemble COVID, they were all assumed to be related to IVM treatment.

\section{Hepatic Safety Analysis}

Although there was a small but statistically significant increase in ALT, AST and total bilirubin values after IVM treatment (Table 3), among 1,022 subjects that were followed up with laboratory determinations after IVM treatment, only one presented liver enzyme values compatible with low grade drug induced liver injury, that lead to drug discontinuation on day 4 of treatment. According to medical records, this subject had abnormal baseline ALT and AST values (AST 240 U/1, ALT 375 $\mathrm{U} / \mathrm{l}$, Total Bilirrubin $0.63 \mathrm{mg} / \mathrm{dl}$ and the values peaked to AST $366 \mathrm{U} / \mathrm{l}$ ALT $630 \mathrm{U} / \mathrm{l}$ total bilirrubin $0.8 \mathrm{mg} / \mathrm{dl}$ and returned to AST $111 \mathrm{U} / \mathrm{l}$, ALT $214 \mathrm{U} / \mathrm{l}$ and total bilirrubin $0.62 \mathrm{mg} / \mathrm{dl}$ ). Considering the total hepatic safety sample $(n=1,022)$, this represents an incidence of $0.98 / 1,000$ treated subjects.

\section{Program's Efficacy}

In order to evaluate the program's efficacy, the clinical evolution of subjects in the IVM-ITT analysis group $(n=3,266)$ was compared with 17,966 subjects that did not participate in the program (C group).
In the whole sample analysis, there was a non-significant tendency toward lower ICU admission in the IVM-ITT group $(28 / 3,266)$ compared with C $(208 / 17,966)$ ( 0.9 vs. $1.2 \%$, odds ratio 0.738 ) (95\% CI $0.497-1.097$, NNT 333, NS). Mortality rate was significantly lower in the IVM-ITT group $(50 / 3,266)$ compared with $C(380 / 17,966)$ (1.5 vs. $2.1 \%$ with an odds ratio of 0.720$)$ (95\% CI 0.535-0.969, NNT 172; $p=0.029$ ) (Figure 2A).

No significant differences were observed in ICU admission (0.4 vs. $0.3 \%$, odds ratio 1.265$)$ (95\% CI $0.484-3.310$, NS) between IVM-ITT group $(5 / 1,415)$ compared with C $(25 / 8,944)$ in subjects younger than 40 year old. Similarly, no significant differences were observed in mortality rate in this age group (IVM-ITT group: $0 / 1,415$ vs. C: $13 / 8,944)$ (0.0 vs. $0.1 \%$, NS) (Figure 2B). Conversely, regarding clinical evolution of subjects $\geq 40$ year old ( $n=9,022$; IVM-ITT $n=1,851)$, ICU admission was significantly lower in the IVM-ITT group $(23 / 1,851)$ compared with C $(183 / 9,022)(1.2$ vs. $2.0 \%$, with an odds ratio of 0.608 ) (95\% CI $0.393-0.940$, NNT 128; $p=0.024$ ). Mortality rate was significantly lower in the IVM-ITT group $(50 / 1,851)$ compared with $\mathrm{C}(367 / 9,022)$ (2.7 vs. $4.1 \%$, with an odds ratio of 0.655 ) (95\% CI 0.485-0.884, NNT 74, $p=0.005$ ) (Figure 2C).

Similar results were observed in the sub-analysis performed after excluding subjects with neoplastic diseases. Specifically, there was a non-significant tendency toward lower ICU admission in the IVM-ITT group $(27 / 3,203)$ compared with C $(202 / 17,697)$ ( 0.8 vs. $1.1 \%$, odds ratio 0.736$)$ (95\% CI $0.492-1.102$, NS) and a lower mortality rate in the IVM-ITT group $(48 / 3,203)$ compared with $C(351 / 17,697)(1.5$ vs. $2.0 \%)$ with an odds ratio of 0.752 (95\% CI $0.555-1.019)(p=0.065)$ in the whole sample analysis. Regarding clinical evolution of subjects $\geq 40$ year old, ICU admission remained significantly lower in the IVM-ITT group $(22 / 1,798)$ compared with C $(177 / 8,781)(1.2$ vs. $2.0 \%$, with an odds ratio of 0.602$)$ (95\% CI $0.385-0.941)(p=0.024)$ after excluding subjects with neoplastic diseases, and mortality rate was significantly lower in the IVM-ITT group $(48 / 1,798)$ compared with $\mathrm{C}(338 / 8,443)(2.7$ vs. $3.8 \%$, with an odds ratio of 0.685 ) (95\% CI 0.504-0.931) ( $p=0.015$ ). No significant differences were observed in ICU admission ( 0.4 vs. $0.3 \%$, odds ratio 1.270 ) (95\% CI $0.485-3.323$, NS) between IVM-ITT group $(5 / 1,405)$ compared with $C(25 / 8,916)$ in subjects younger than 40 year old). Similarly, no significant differences were observed in mortality rate in this age group (IVM-ITT group: 0/1,415 vs. C: $13 / 8,916)(0.0$ vs. $0.1 \%$, NS).

In the analysis of all non-immunized subjects ( $\mathrm{C} n=16,226$; IVM: $n=2,936)$, there was a non-significant tendency toward lower ICU admission in the IVM-ITT group $(21 / 2,936)$ compared with C $(181 / 16,226)(0.7$ vs. $1.1 \%$, odds ratio 0.639$)$ (95\% CI $0.406-1.005$, NNT 250; $p=0.051)$. Mortality rate was significantly lower in the IVM-ITT group $(32 / 2,936)$ compared with C $(280 / 16,226)$ (1.1 vs. $1.7 \%$, with an odds ratio of 0.628$)$ (95\% CI 0.434-0.907, NNT 158; $p=0.012$ ).

No significant differences were observed in ICU admission ( 0.4 vs. $0.3 \%$, odds ratio 1.271 ) (95\% CI $0.486-3.326$, NS) between IVM-ITT group $(5 / 1,380)$ compared with C $(25 / 8,763)$ in nonimmunized subjects younger than 40 year old, Similarly, no significant differences were observed in mortality rate between 
A

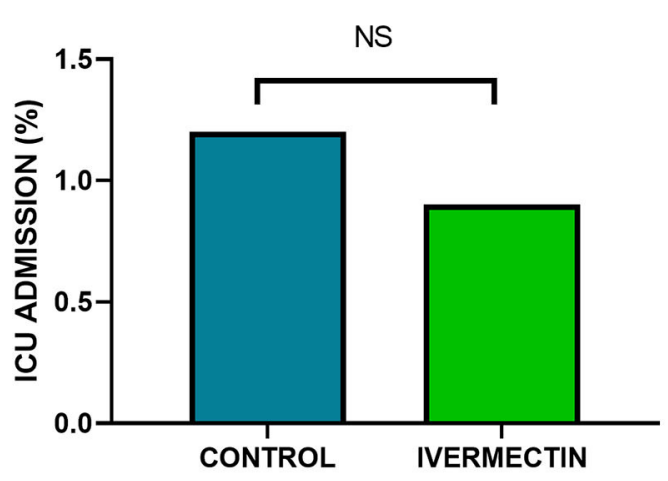

B

\section{Subjects between 18 and 40 year-old}
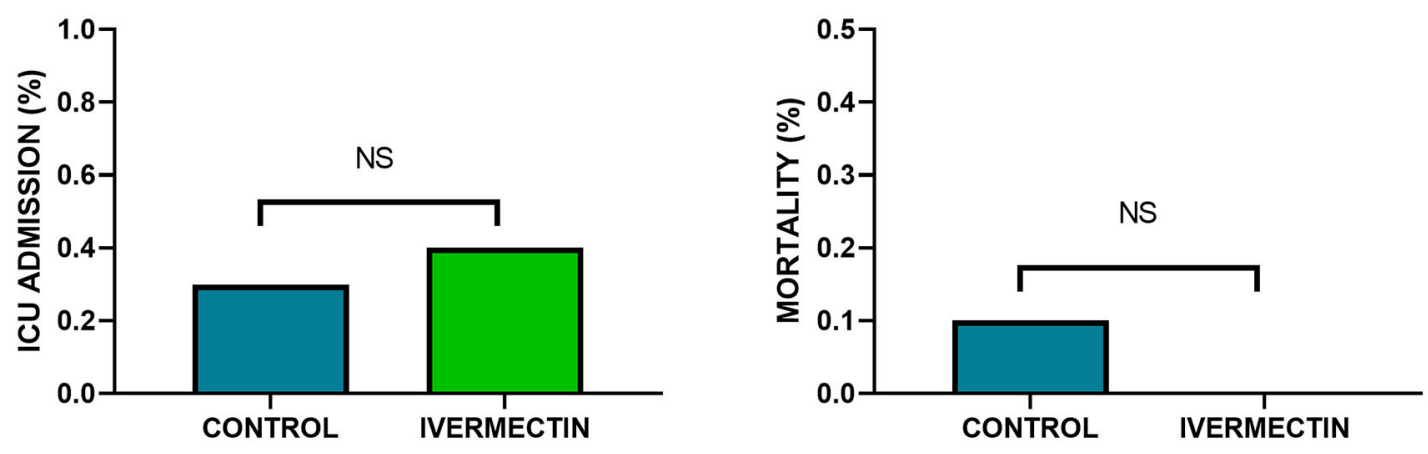

C

Subjects $\geq \mathbf{4 0}$ year-old
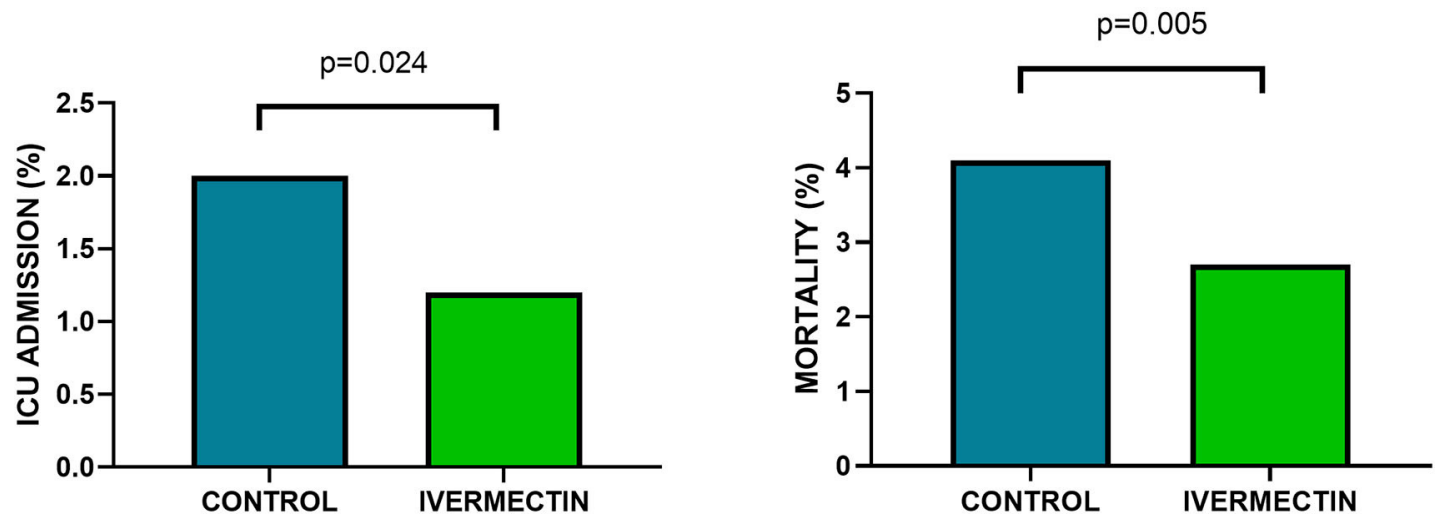

FIGURE 2 | ICU admission and mortality in IVM-ITT and C groups. (A) Whole population: analysis of all subjects (C $n=17,966$; IVM-ITT $n=3,266) ;$ (B) Analysis of subjects $\geq 18$ year-old and $<40$ year-old (C $n=8,944$; IVM-ITT $n=1,415)$; (C) Analysis of subjects $\geq 40$ year-old $(\mathrm{C} n=9,022$; IVM-ITT $n=1,851)$. NS, non statistically significant; $n$, number of subjects. 
non-immunized subjects younger than 40 year old (IVM-ITT group: $0 / 1,380$ vs. C: $13 / 8,763)(0.0$ vs. $0.1 \%$, NS).

Conversely, regarding clinical evolution of non-immunized subjects $\geq 40$ year old (C $n=7,463$ and IVM-ITT $n=1,556$ ), ICU admission was significantly lower in the IVM-ITT group $(16 / 1,556)$ compared with C $(156 / 7,463)$ (1.0 vs. $2.1 \%$, with an odds ratio of 0.487 ) (95\% CI $0.290-0.816$, NNT 95; $p=0.005)$. Mortality rate was significantly lower in the IVM-ITT group $(32 / 1,556)$ compared with C $(267 / 7,463)$ (2.1 vs. $3.6 \%)$, with an odds ratio of 0.566 (95\% CI $0.391-0.820$, NNT $66 ; p=0.002$ ).

A total of 2,895 subjects in the IVM group had complete data regarding weight-based ivermectin dose information (of 3,266 subjects assigned to IVM, 146 had missing data regarding the prescribed dose, and 335 had missing body weight data).

Mean \pm SD IVM prescribed dose was $44.15 \pm 11.83 \mathrm{mg} /$ day, mean IVM prescribed dose per $\mathrm{kg}$ of body weight was $0.54 \pm$ $0.09 \mathrm{mg} / \mathrm{kg} /$ day. Based on these values, two IVM groups were created: Low-dose IVM (with prescribed dose lower than 0.54 $\mathrm{mg} / \mathrm{kg} /$ day, $n=1,157$ ) and High-dose IVM (with a prescribed dose equal or higher than $0.54 \mathrm{mg} / \mathrm{kg} /$ day, $n=1,738)$. No significant differences were observed between groups regarding descriptive characteristics (age, gender, immunization status and comorbidities; data not shown). As expected, IVM prescribed dose per $\mathrm{kg}$ of body weight was significantly higher in the High-dose IVM group (mean \pm SD: $0.597 \pm 0.067 \mathrm{mg} / \mathrm{kg} /$ day) compared to the Low-dose IVM group (mean \pm SD: $0.459 \pm$ $0.079 \mathrm{mg} / \mathrm{kg} /$ day) $(p<0.001)$.

In the whole sample analysis of High-dose IVM vs. Low-dose IVM, there was a non-significant tendency toward lower ICU admission in the High-dose IVM group compared with Lowdose IVM (0.6 vs. $1.2 \%)$, with an odds ratio of 0.472 (95\% CI $0.209-1.067 ; p=0.065)$. There were no significant differences in mortality rate ( 1.2 vs. $1.6 \%$ odds ratio of 0.733$)$ (95\% CI $0.392-1.369$; NS).

Regarding clinical evolution of subjects with an age of $\geq$ 40 year old (Low-dose IVM $n=645$ and High-dose IVM $n=1,016)$, there was a non-significant tendency toward lower ICU admission in the High-dose IVM group ( 0.8 vs. $1.7 \%$ with an odds ratio of 0.457 ) (95\% CI $0.183-1.143 ; p=0.086)$. There were no significant differences in mortality rate $(2.1 \mathrm{vs.} 2.9 \%$ odds ratio of 0.695 ) (95\% CI 0.371-1.304; NS).

In the analysis of all subjects receiving High-dose IVM $(n=1,738)$ vs. $\mathrm{C}(n=17,966)$, ICU admission was significantly lower in the High-dose IVM group compared with C (0.6 vs. $1.2 \%)$, with an odds ratio of 0.494 (95\% CI 0.261-0.934, NNT 172; $p=0.027)$ and mortality rate was lower in the High-dose IVM group compared with C (1.2 vs. $2.1 \%$ odds ratio of 0.566$)(95 \%$ CI $0.364-0.881$, NNT $111 ; p=0.01)$. Similarly, simple logistic regression analysis of ICU admission and mortality rate in Highdose IVM, Low-dose IVM and C showed that meanwhile there were no significant differences in ICU admission (exponential $B$ $1.046,95 \%$ CI $0.607-1.802$; NS) or mortality rate (exponential B 0.773 , 95\% CI $0.486-1.230$; NS) between Low-dose IVM compared to C, High-dose IVM was associated with lower ICU admission (exponential $B$ 0.494, 95\% CI 0.261-0.934; $p=0.03$ ) and mortality rate (exponential $B \quad 0.566,95 \%$ CI $0.364-0.881$; $p=0.012$ ) compared with $\mathrm{C}$. No significant differences were observed between groups regarding ICU admission and mortality rate in subjects younger than 40 year old (data not shown).

Regarding clinical evolution of subjects with an age of $\geq 40$ year old (C $n=9,212$; IVM-ITT $n=1,016$ ), ICU admission was significantly lower in the High-dose IVM group compared with C (0.8 vs. $2.0 \%)$, with an odds ratio of 0.383 (95\% CI $0.188-0.780$, NNT $81 ; p=0.006)$. Similarly, mortality rate was lower in the High-dose IVM group compared with $\mathrm{C}(2.1$ vs. $4.1 \%$ odds ratio of 0.498 ) (95\% CI $0.319-0.776$, NNT 50; $p=0.002$ ). Simple logistic regression analysis of ICU admission and mortality rate in High-dose IVM, Low-dose IVM and C showed that meanwhile there were no significant differences in ICU admission (exponential B 0.838, 95\% CI 0.454-1.548; NS) or mortality rate (exponential $B \quad 0.716,95 \%$ CI $0.448-1.143$; NS) between Low-dose IVM compared to C, High-dose IVM was associated with lower ICU admission (exponential B 0.383, 95\% CI $0.188-0.780 ; p=0.008$ ) and mortality rate (exponential $B$ $0.498,95 \%$ CI $0.319-0.776 ; p=0.002$ ) compared with C.

A logistic regression analysis was performed after adjusting for sex, age, immunization status and comorbidities, and IVM treatment remained negatively associated with ICU admission rate and mortality rate (Figure 3 ).

\section{DISCUSSION}

This report of a monitored intervention program with ivermectin in COVID-19 patients provides observational data on a significant number of adult patients that through the incorporation of clinical and demographic data from a large number of patients from the same province and period but not participating in the program (control group) allowed a comparison and analysis of hard clinical endpoints as are admission to ICU and death. This comparison provides results that suggest a significant positive clinical impact of this intervention that, in the context of a lack of proven antiviral alternative treatments for ambulatory patients against COVID19, the safety, availability and affordability of IVM and the growing concerns on vaccine efficacy against emerging variants of SARS-CoV-2, deserves consideration as a potential tool for case management.

The approach taken in the Province of La Pampa for the use of IVM through a MEURI Program supported and leaded by the provincial Ministry of Health was based on preliminary but inconclusive data on efficacy and a more solid reference base on the safety of the drug, even at higher doses than those approved for other indications in Argentina, as strongyloidiasis and scabies (23). The selection of high-doses of IVM was based on the results of the in-vitro reports on the antiviral activity of IVM against SARS-CoV-2 as well as the growing body of evidence on the safety of these higher than usual doses for other indications in pediatric and adult populations (23-25). With the incorporation of over three thousand cases that completed treatment and follow up, including 1022 with clinical laboratory monitoring, this intervention program contributes the largest analysis on the safety of high dose ivermectin at a regimen of $600 \mu \mathrm{g} / \mathrm{kg} / \mathrm{day}$ for 


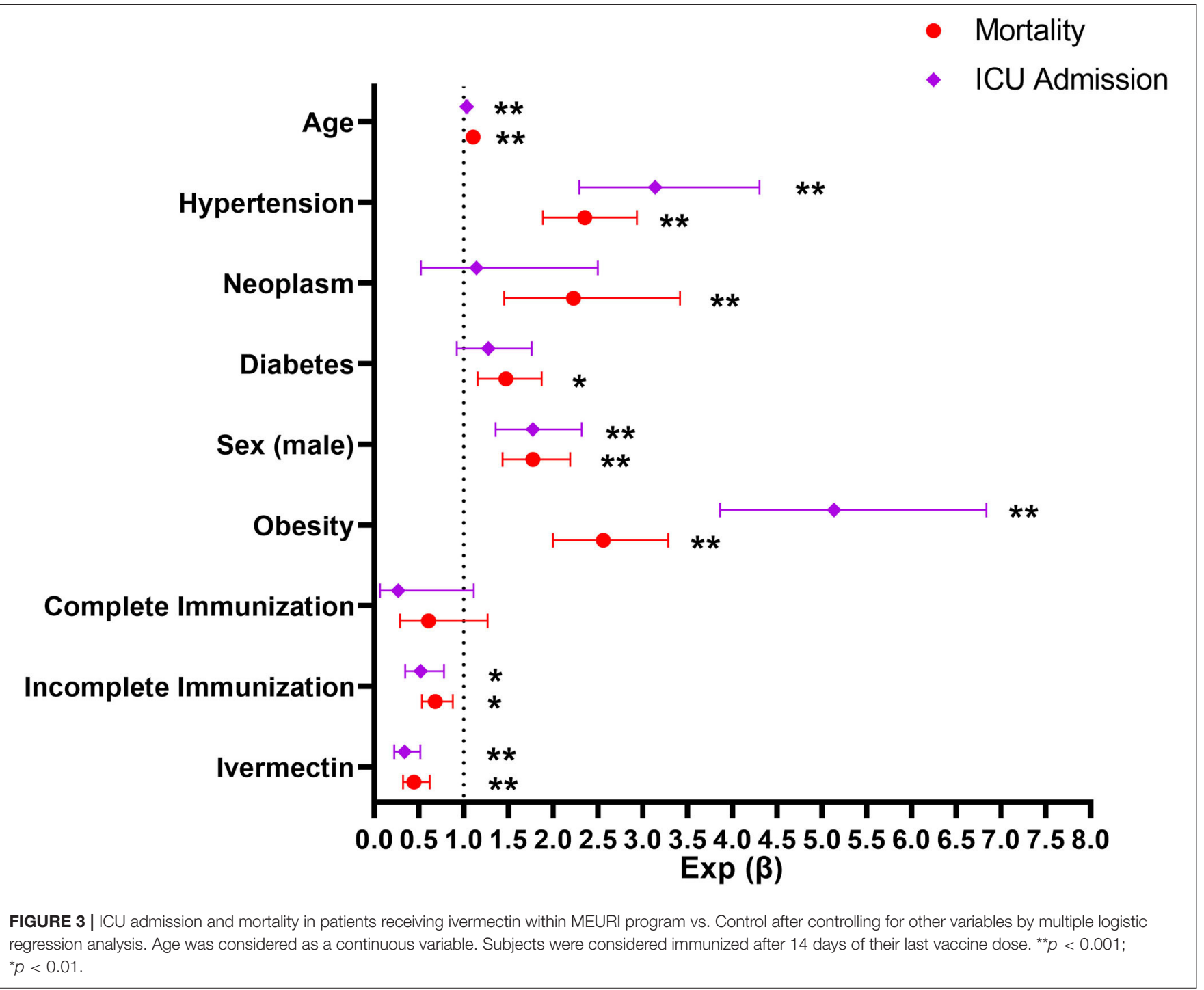

5 consecutive days. This regimen was selected based on a proofof-concept trial that identified a significant antiviral activity against SARS-CoV-2 in a subgroup of participant who achieved high IVM median plasma concentrations without any significant safety issues (17). In view of that seemingly dose response antiviral effect, participants of our program were advised to ingest the daily doses of IVM after a meal with adequate fat content in view of the lipophilic nature of $\operatorname{IVM}(13,26)$. The favorable safety profile of this regimen of high dose IVM, which includes the identification of adverse events that were comparable in type and severity to those reported in a meta-analysis on the safety of high-dose IVM (23), in a setting with conditions for high oral bioavailability, confirms prior communications with smaller sample sizes, on the safety of these regimens and allows to focus in exploring the clinical efficacy of these regimens for a variety of clinical indications for which IVM is in pre-clinical and clinical development as a repurposed drug including COVID-19, dengue, trichuriasis and malaria. The finding of a single case of clinically significant increase in liver enzymes, in an individual with baseline abnormal values highlights these clinical and laboratory findings and provides evidence for the design of simplified MEURI Programs, should they be considered appropriate.

The controversial findings around the efficacy of IVM in COVID-19 is currently preventing from making firm recommendations to clinicians. The situation is worsened by confusing messages through social and traditional media plus articles from peer-reviewed journals that are published and afterwards retracted as well as the uncontrolled use of medical and veterinary products by the population (27). Metaanalyses that included studies with a variety of regimens have reached different interpretations and conclusions, preventing the achievement of consistent findings $(14,18,28,29)$. In this context, a MEURI Program appears as the mean to attempt monitored and controlled use of a treatment not approved for the indication but with preliminary results of adequate safety and potential efficacy. While the impossibility to perform clinical trials was not an absolute situation at the time of the design of our Program, the means and capacities of a provincial Ministry of Health were beyond the scope and resources available. 
The role of IVM against SARS-CoV-2 was supported by the biologic plausibility based on in vitro and in vivo studies of mechanistic analyses and antiviral effects. Its proposed antiviral mechanism is thought to be mediated by its ability to inhibit the nuclear import of viral proteins mediated by IMPa/b1 heterodimer, and the promotion of defense mechanisms such as pyroptosis in infected epithelial cells, suggesting its possible role as a broad spectrum antiviral agent $(30,31)$. Although an immunomodulatory effect of IVM has been proposed by other authors (32), these effects might explain the antiviral activity against SARS-CoV-2 reported by our group and Biber et al., in two small randomized controlled trials $(17,19)$. However, randomized clinical trials published on this topic have shown a lack of efficacy regarding clinical outcomes. Lopez Medina et al. reported on the failure to show a significant effect of $300 \mu \mathrm{g} / \mathrm{kg}$ of IVM for 5 consecutive days vs. placebo in symptom's resolution among 400 patients with mild disease recruited during the first week of COVID-19 in a single center in Colombia (15). This trial, that was originally designed to demonstrate improvement of 2 points in WHO Ordinary Scale but suffered from fewer than estimated events, included a population with a median age of 37 year-old and administered IVM on an empty stomach, as indicated by the manufacturer, which probably prevented from maximizing oral biovailability of this highly lipophilic drug. In the study from Vallejos et al. in Argentina (16), 501 patients with mild early COVID-19 infection were randomized to receive placebo or IVM for 2 consecutive days at up to $200 \mu \mathrm{g} / \mathrm{kg}$ (the currently approved dose for other indications). Neither hospitalization (primary outcome) nor other secondary outcomes including polymerase chain reaction test negativity and safety outcomes showed statistically significant differences between groups in this population with fewer events than estimated a priori and a mean age of 42 year-old ( $S D \pm 15.5)$.

The seemingly contrasting efficacy results between our analysis of an intervention program vs. the double-blind, placebo-controlled RCTs might not be discordant at closer look with reasons laying in several factors pertaining to IVM regimens, population size and outcomes; but potential bias in our results is another possibility to be considered and explored. The IVM regimen used in our program provided a higher total exposure to the drug through a higher dose per day, longer treatment (compared to the trial by Vallejos) and administration with food, which while equally safe might have allowed reaching drug levels at the relevant tissues above the threshold required for an antiviral activity resulting in better clinical outcomes (16). In terms of the populations included in the analyses, it is relevant to consider that both RCTs reached fewer primary endpoints than estimated for the sample size calculation; notwithstanding those potential limitations, which might have been affected by the age of the population recruited to those trials, neither adjustments nor secondary outcomes identified any significant clinical findings. Despite sound trial methodology, failure to demonstrate the effect of an intervention might reside in contextual elements as is the recruitment of subjects at very low risk of achieving the primary outcome regardless of the use of an intervention $(33,34)$, as might have been the case in both RCTs as well as our observational analysis, which due to the significantly larger population size, was able to identify a statistically significant treatment effect in admission to ICU and death in restricting the analysis to those $>40$ year-old.

Statistically significant differences in observational studies should be viewed with caution since the clinical and public health relevance of those results might not be judged as relevant despite the statistics. Based on that, NNT ratios provide an indicator that could inform clinicians and policy makers on the value and convenience of this intervention. Effect size is another element to be considered in the evaluation of an intervention, since the ability of trials to rule-out the effect of an intervention grows in the required sample size in direct relationship to the decrease in the effect size, with direct implications in the feasibility of a clinical trial and the convenience of an intervention (35). In the present analysis, as expected, NNT related to ICU admission and mortality prevention differed significantly between subgroups, with a lower NNT in higher risk groups, as subjects older than 40 year-old.

As an observational intervention, our analyses are susceptible to bias, which constitute the most significant limitation of this report and a major concern in COVID-19 in view of the multitude of publications of studies and observations designed, run and published at unprecedented speed (36). The risk of confounding factors introducing bias in the comparison between groups cannot be completely ruled out, although several measures were taken to minimize its occurrence, like the verification of balanced age distribution, vaccination status and prevalence of comorbidities, with a special attention paid to current oncologic processes that could identify patients with terminal disease. In reference to it, in a sub-analysis that excluded individuals with current neoplams the differences between IVM and C groups remained significant. Survivor bias was assessed and controlled in a sub-analysis (data not shown) through the exclusion from the analysis of all individuals in the control groups whose death occurred within the first 4 days since diagnosis, since those individuals were in all likelihood not offered the intervention, maintaining a significant association in favor of IVM in terms of mortality frequency in the higher risk groups (non-immunized subjects older than 40 year-old). In order to limit the mortality assessment to death related to COVID-19 in the C group, deaths were only considered for the analysis when occurring during the original hospital admission or within a month after discharge. Regarding the possible influence of differences in the prevalence of comorbidities between groups, it is important to highlight that, meanwhile most comorbidities were balanced between groups, a higher percentage of participants in the IVM group reported hypertension and obesity compared to C. Although these differences could be attributed to methodological differences in the identification of comorbidities, should they be real, describe a higher risk of disease progression and, consequently, would not explain the better outcomes observed in this group.

A per-protocol analysis including only individuals that completed the whole treatment, which could provide information of the full potential of the regimen was not performed since individuals on therapy that had their treatments interrupted at hospital admission were identified and given 
that situation, performing a per-protocol analysis would have given biased results that would wrongfully inflate the efficacy of the intervention.

One limitation of the present work is the absence of a detailed record of the refusal of patients to participate in the IVM program. Specifically, since the start of the program in January 2021 , every physician in the province was authorized to offer this treatment to patients who met the inclusion criteria. However, the inclusion of patients was left to the discretion of the treating physician and to the acceptance by the patient. For this reason, the non-inclusion of patients in the IVM program could be due both to the refusal of patients to receive treatment after it was offered, to non-compliance with the inclusion criteria, or to the decision by the treating physician not to offer this treatment option. Unfortunately, no information is available to confirm the reason for not including each individual patient. Another limitation is the lack of pharmacokinetic data to identify a relationship between drug levels and clinical findings, which was beyond the scope of the Program.

When looking at our findings in the context of the results and conclusions of rigorous RCTs, other observational clinical studies, virologic and molecular biology studies for the evaluation of IVM in COVID-19, we conclude on the plausibility and potential clinical utility of IVM at higher doses than currently approved, optimizing its bioavailability but with a relatively moderate effect size in high-risk population groups. Without safety concerns at the doses used in our program, this report highlights IVM as an intervention that deserves a dispassionate, careful, public health-based consideration for the treatment of patients during present COVID-19 pandemic until superior therapeutic alternatives become available and affordable, and highlights the importance of performing adequately powered RCTs in order to confirm our findings. Comparisons between repurposed molecules like IVM vs. newly developed drugs like molnupiravir, tixegevimab-cilgavimab or remdesivir in terms of safety, efficacy, cost and availability will help identifying the potential role of each of these commercially available drugs for the management of COVID-19 patients.

\section{DATA AVAILABILITY STATEMENT}

The raw data supporting the conclusions of this article will be made available by the authors, without undue reservation.

\section{ETHICS STATEMENT}

The studies involving human participants were reviewed and approved by the Provincial Ethics Committee of La Pampa, and participating individuals provided written informed consent. The patients/participants provided their written informed consent to participate in this study.

\section{AUTHOR CONTRIBUTIONS}

$\mathrm{AF}, \mathrm{MB}, \mathrm{AP}, \mathrm{JB}, \mathrm{CD}, \mathrm{MM}, \mathrm{MAM}, \mathrm{AK}, \mathrm{GV}$, and $\mathrm{MK}$ : conceptualization. AF, MB, CE, AP, JB, CD, MM, and JO: data curation. MAM, AK, PF, AF, and MK: formal analysis. MAM, AK, $\mathrm{AF}, \mathrm{MB}, \mathrm{JB}, \mathrm{MM}, \mathrm{AP}, \mathrm{CD}, \mathrm{CE}, \mathrm{AB}, \mathrm{GV}$, and $\mathrm{MK}$ : investigation. AF, MB, JB, MM, AP, CD, and MK: methodology. MAM, GV, and $\mathrm{MK}$ : supervisión. MAM, AK, and $\mathrm{MK}$ : writing-original draft. MAM, AK, AF, MB, JB, MM, AP, CD, CE, AB, PF, GV, and $M K$ : writing-review and editing. All authors contributed to the article and approved the submitted version.

\section{FUNDING}

This work was supported by the Government of La Pampa, Argentina.

\section{ACKNOWLEDGMENTS}

The authors would like to acknowledge the authorities of the Government of the Province of La Pampa, Argentina, for their support, and to the Ivermectin Monitored Intervention program collaborators: María Alejandra Ramallo; Andrea Barbero; Liliana Torres; Nadia Paola Andresco Soto; Lucía Martín; Analisa Beneitez; Daniela Fischman; Marcela Natividad Millagueque; Carolina Andrea Spallina; Leandro Ceferino Deambrossio; Silvina Ramos Monso; Emiliano Renzo Beraudo; Raúl Javier Gavotti; Noelia del Valle Godino; Nora Ester Bertón; Carla Noelia Morales; Mariné del Valle Plizzo; Héctor Marsollier; María Estela Martin, Eugenia María Tabares, Lesly Godoy; Gerardo Minudri; Lourdes Bramante; Natalia Mendoza; Paloma De la Prida; María Soledad Arambarry; Silvia Lluch; Vanesa Garro; Santiago Arisi; Silvia Kredes; Gisela Bissio; Marcelo Allara; Juan Guerrero; Pablo Gutierrez; Pamela Astesano, Natalia Baez; Pamela Ulivetti; Mariano Quiroga; Romina Londero; Lorena Carasay; Diego Arroyo; Florencia Calo; Ruth Villagra; Marianela Barbero; Betina Larroque; Angihomar Hernandez; Maria Julia Luján; Enrique Quiroga; Silvia Romera; José Alfonso; Gastón Verna; Nancy Jouly; Gustavo López; Gabriela Barra; Astrid Pérez; Darío Bustos; Yanina Monarde, Dafne Rojas; Franco Balsa; Paola Cervellini, Ariel Soto; Silvia Soto; Cesar Sosa; Bibiana Widemman; Adolfo Barrionuevo; Cecilia Schneider; Adrián Díaz, Luisa Macayo; Ludmila Stern; Maximiliano Elias; Fernando Andreatta; Juleth Mercado: Horacio Ortiz; Oscar Mas; Marcelo Carrizo; Ludvina Luft; María Barrionuevo; Liliana Burgahrtd, Mariana Puglie; Jessica Puglie; María Naab; Carolina De los santos; Ruben Solett; Rocío Cerrada; Carolina Redel; Gloria Baigorria; Gustavo Topfanini; Norberto Rovera; María Morales; Nora Delfino; Amalia León; Alejandra Torres; Matías Muria; Clarisa Gómez; Jorge Jorja; Daniel Celi; Franco Saini, Natalia Vago; Patricia Gimenez; María Torres; Matías Altamira; María Juri, Noelia Rosa; Rita Guerbilli; Pamela Leguizamon; Jorge García; Mariela Torres; Daniela Anechi; Cristian González; Miguel Hermua; Silvina Furik; Noelia Baizero; Silvia Moran; Cecilia Abdala; Erika Yoshida; Anabella Penego; Agustina Abdala; Carla Barboza; Natalia Maison; Rosa Sepulveda; Alicia Cappozi; Vanesa Ferreyra; Yanina Verdugo; Daiana Vanderberg; Dayana Iglesias; Maylen Aimetta; Pablo Sosa; Joaquín Cepeda; Gustavo Sosa; Lilia Schan; Laura Galarza. 


\section{REFERENCES}

1. Wu F, Zhao S, Yu B, Chen Y-M, Wang W, Song Z-G, et al. A new coronavirus associated with human respiratory disease in China. Nature. (2020) 579:2659. doi: 10.1038/s41586-020-2008-3

2. Johns Hopkins University. COVID-19 Dashboard. Available online at: https://gisanddata.maps.arcgis.com/apps/opsdashboard/index.html\#/ 6f1ed23bcae049af84a3d23ef2201510 (accessed January 26, 2022).

3. Moline HL, Whitaker M, Deng L, Rhodes JC, Milucky J, Pham H, et al. Effectiveness of COVID-19 vaccines in preventing hospitalization among adults aged $\geq 65$ years - COVID-NET, 13 states, February-April (2021). Morb Mortal Wkly Rep. (2021) 70:1088-93. doi: 10.15585/mmwr.mm7032e3

4. Thompson MG, Stenehjem E, Grannis S, Ball SW, Naleway AL, Ong TC, et al. Effectiveness of Covid-19 vaccines in ambulatory and inpatient care settings. N Engl J Med. (2021) 7:1355-71. doi: 10.1056/NEJMoa2110362

5. Jerving S. The long road ahead for COVID-19 vaccination in Africa. Lancet. (2021) 398:827-8. doi: 10.1016/S0140-6736(21)01967-X

6. National Institutes of Health. COVID-19 Treatment govGuidelines Panel. Coronavirus Disease 2019 (COVID-19) Treatment Guidelines. (2021). Available online at: https://www.covid19treatmentguidelines.nih.gov (accessed January 26, 2022).

7. Siemieniuk RAC, Bartoszko JJ, Ge L, Zeraatkar D, Izcovich A, PardoHernandez H, et al. Drug treatments for covid-19: living systematic review and network meta-Analysis. BMJ. (2020) 370:m2980. doi: 10.1136/bmj. $\mathrm{m} 2980$

8. COVID-19 Treatment Guidelines Panel. Coronavirus Disease 2019 (COVID19) Treatment Guidelines. National Institutes of Health. Available online at https://www.covid19treatmentguidelines.nih.gov (accessed January 24, 2022).

9. Omura S, Crump A. Ivermectin: panacea for resource-poor communities? Trends Parasitol. (2014) 30:445-55. doi: 10.1016/j.pt.2014.07.005

10. Thylefors B, Alleman MM, Twum-Danso NAY. Operational lessons from 20 years of the Mectizan Donation Program for the control of onchocerciasis. Trop Med Int Heal. (2008) 13:689-96. doi: 10.1111/j.1365-3156.2008.02049.x

11. Laing R, Gillan V, Devaney E. Ivermectin - old drug, new tricks? Trends Parasitol. (2017) 33:463-72. doi: 10.1016/j.pt.2017.02.004

12. Suputtamongkol Y, Avirutnan P, Mairiang D, Angkasekwinai N, Niwattayakul $\mathrm{K}$, Yamasmith E, et al. Ivermectin accelerates circulating nonstructural protein 1 (NS1) clearance in adult dengue patients: a combined phase $2 / 3$ randomized double-blinded placebo controlled trial. Clin Infect Dis an Off Publ Infect Dis Soc Am. (2021) 72:e586-93. doi: 10.1093/cid/ciaa1332

13. Caly L, Druce JD, Catton MG, Jans DA, Wagstaff KM. The FDA-approved drug ivermectin inhibits the replication of SARS-CoV-2 in vitro. Antivir Res. (2020) 178:104787. doi: 10.1016/j.antiviral.2020.104787

14. Padhi S, Pati A, Panda AK. Effect of ivermectin in the treatment of COVID-19: a trial sequential analysis highlighted the requirement of additional randomized controlled trials. Clin Infect Dis. (2021) ciab692. doi: 10.1093/cid/ciab692

15. López-Medina E, López P, Hurtado IC, Dávalos DM, Ramirez O, Martínez E, et al. Effect of ivermectin on time to resolution of symptoms among adults with mild COVID-19: a randomized clinical trial. J Am Med Assoc. (2021) 325:1426-35. doi: 10.1001/jama.2021.3071

16. Vallejos J, Zoni R, Bangher M, Villamandos S, Bobadilla A, Plano F, et al. Ivermectin to prevent hospitalizations in patients with COVID-19 (IVERCOR-COVID19) a randomized, double-blind, placebo-controlled trial. BMC Infect Dis. (2021) 21:1-11. doi: 10.1186/s12879-02106348-5

17. Krolewiecki A, Lifschitz A, Moragas M, Travacio M, Valentini R, Alonso DF, et al. Antiviral effect of high-dose ivermectin in adults with COVID-19: a proof-of-concept randomized trial. EClinicalMedicine. (2021) 37:e100959. doi: 10.1016/j.eclinm.2021.100959

18. Bryant A, Lawrie TA, Dowswell T, Fordham EJ, Mitchell S, Hill SR, et al. Ivermectin for prevention and treatment of COVID-19 infection: a systematic review, meta-analysis, and trial sequential analysis to inform clinical guidelines. Am J Ther. (2021) 28:e434-60. doi: 10.1097/MJT.0000000000001442

19. Biber A, Mandelboim M, Harmelin G, Lev D, Ram L, Shaham A, et al. Favorable outcome on viral load and culture viability using Ivermectin in early treatment of non-hospitalized patients with mild
COVID-19 - A double-blind, randomized placebo-controlled trial. medRxiv. (2021). doi: 10.1101/2021.05.31.21258081

20. Chaccour C, Casellas A, Blanco-Di Matteo A, Pineda A, Fernandez-Montero A, Ruiz-Castillo $\mathrm{P}$, et al. The effect of early treatment with ivermectin on viral load, symptoms and humoral response in patients with nonsevere COVID-19: a pilot, double-blind, placebo-controlled, randomized clinical trial. EClinicalMedicine. (2021) 32:100720. doi: 10.1016/j.eclinm.2020. 100720

21. World Health Organization (WHO). Ethical issues related to study design for trials on therapeutics for Ebola Virus Disease. WHO Ethics Work Gr Meet. (2014) 1-7.

22. Bessone F, Hernandez N, Tagle M, Arrese M, Parana R, Méndez-Sánchez $\mathrm{N}$, et al. Drug-induced liver injury: a management position paper from the Latin American Association for Study of the liver. Ann Hepatol. (2021) 24:100321. doi: 10.1016/j.aohep.2021.100321

23. Navarro M, Camprubí D, Requena-Méndez A, Buonfrate D, Giorli G, Kamgno J, et al. Safety of high-dose ivermectin: a systematic review and metaanalysis. J Antimicrob Chemother. (2020) 75:827-34. doi: 10.1093/jac/dk z524

24. Smit MR, Ochomo EO, Aljayyoussi G, Kwambai TK, Abong’o BO, Chen T, et al. Safety and mosquitocidal efficacy of high-dose ivermectin when co-administered with dihydroartemisinin-piperaquine in Kenyan adults with uncomplicated malaria (IVERMAL): a randomised, double-blind, placebo-controlled trial. Lancet Infect Dis. (2018) 18:615-26. doi: 10.1016/S1473-3099(18)30163-4

25. Matamoros G, Sanchez A, Gabrie JA, Juarez M, Ceballos L, Escalada, et al. Efficacy and safety of albendazole and high-dose ivermectin coadministration in schoolaged children infected with Trichuris trichiura in Honduras: a randomized controlled trial. Clin Infect Dis. (2021) 73:120310. doi: $10.1093 / \mathrm{cid} / \mathrm{ciab} 365$

26. Edwards G, Dingsdale A, Helsby N, Orme MLE, Breckenridge AM. The relative systemic availability of ivermectin after administration as capsule, tablet, and oral solution. Eur J Clin Pharmacol. (1988) 35:6814. doi: $10.1007 / \mathrm{BF} 00637608$

27. Hill A, Garratt A, Levi J, Falconer J, Ellis L, McCann K, et al. Expression of concern: "meta-analysis of randomized trials of ivermectin to treat SARS-CoV-2 infection." Open Forum Infect Dis. (2021) 8:ofab394. doi: 10.1093/ofid/ofab394

28. Roman YM, Burela PA, Pasupuleti V, Piscoya A, Vidal JE, Hernandez AV. Ivermectin for the treatment of COVID-19: a systematic review and meta-analysis of randomized controlled trials. Clin Infect Dis. (2021). doi: 10.1101/2021.05.21.21257595

29. Neil M, Fenton N, Kow CS, Hasan SS, Popp M, Stegemann M, et al. Bayesian hypothesis testing and hierarchical modeling of ivermectin effectiveness. Am J Ther. (2021) 28:CD015017. doi: 10.1097/MJT.00000000000 01450

30. Segatori VI, Garona J, Caligiuri LG, Bizzotto J, Lavignolle R, Toro A, et al. Effect of ivermectin and atorvastatin on nuclear localization of importin alpha and drug target expression profiling in host cells from nasopharyngeal swabs of SARS-CoV-2- positive patients. Viruses. (2021) 13:2084. doi: 10.3390/v13102084

31. Heidary F, Gharebaghi R. Ivermectin: a systematic review from antiviral effects to COVID-19 complementary regimen. J Antibiot. (2020) 72:593602. doi: 10.1038/s41429-020-0336-Z

32. Sajid MS, Iqbal Z, Muhammad G, Iqbal MU. Immunomodulatory effect of various anti-parasitics: a review. Parasitology. (2006) 132:30113. doi: $10.1017 /$ S0031182005009108

33. Manary M. It's the context! Am J Clin Nutr. (2015) 101:6934. doi: 10.3945/ajcn.115.108399

34. Kow CS, Hasan SS. Pitfalls in reporting sample size calculation across randomized controlled trials involving ivermectin for the treatment of COVID-19. Am J Ther. (2021) 28:e616-9. doi: 10.1097/MJT.00000000000 01441

35. Fritz CO, Morris PE, Richler JJ. Effect size estimates: current use, calculations, and interpretation. J Exp Psychol Gen. (2012) 141:2-18. doi: 10.1037/a00 24338

36. Tleyjeh IM, Kashour T, Mandrekar J, Petitti DB. Overlooked shortcomings of observational studies of interventions in coronavirus disease 2019: an 
illustrated review for the clinician. Open Forum Infect Dis. (2021) 8:18. doi: 10.1093/ofid/ofab317

Conflict of Interest: The authors declare that the research was conducted in the absence of any commercial or financial relationships that could be construed as a potential conflict of interest.

Publisher's Note: All claims expressed in this article are solely those of the authors and do not necessarily represent those of their affiliated organizations, or those of the publisher, the editors and the reviewers. Any product that may be evaluated in this article, or claim that may be made by its manufacturer, is not guaranteed or endorsed by the publisher.

Copyright (C) 2022 Mayer, Krolewiecki, Ferrero, Bocchio, Barbero, Miguel, Paladini, Delgado, Ojeda, Elorza, Bertone, Fleitas, Vera and Kohan. This is an open-access article distributed under the terms of the Creative Commons Attribution License (CC $B Y)$. The use, distribution or reproduction in other forums is permitted, provided the original author(s) and the copyright owner(s) are credited and that the original publication in this journal is cited, in accordance with accepted academic practice. No use, distribution or reproduction is permitted which does not comply with these terms. 